\title{
Repeated Implantation is a More Effective Cell Delivery Method in Skeletal Myoblast Transplantation for Rat Myocardial Infarction
}

\author{
Goditha U. Premaratne, MD; Keiichi Tambara, MD; Masatoshi Fujita, MD*; Xue Lin, MD; \\ Naoki Kanemitsu, MD; Shinji Tomita, MD**; Genichi Sakaguchi, MD; \\ Hiroyuki Nakajima, MD; Tadashi Ikeda, MD; Masashi Komeda, MD
}

\begin{abstract}
Background Several clinical trials are underway to determine whether autologous skeletal myoblast transplantation is an effective and safe therapeutic strategy for severe heart failure due to myocardial infarction (MI). It remains unclear whether repeated skeletal myoblast implantation is a feasible and effective cell delivery method for the infarcted myocardium.

Methods and Results Four weeks after a coronary ligation, male syngeneic Lewis rats were assigned to 3 treatment groups: 3 episodes of skeletal myoblasts $\left(6 \times 10^{6}\right)$ transplantation (group I), a bolus transplantation of myoblasts $\left(18 \times 10^{6}\right)$ (group II), or culture medium injection (group III). Eight weeks after the first treatment, echocardiography, cardiac catheterization and histological examination were performed to compare the therapeutic effects on left ventricular (LV) systolic and diastolic functions, and the engrafted myoblast volume. Repeated myoblast implantation significantly improved LV function and resulted in significantly larger engrafted volume and LV contractility compared with a bolus transplantation with the same number of myoblasts.

Conclusions Repeated skeletal myoblast transplantation is a safe and effective therapeutic strategy for the infarcted myocardium. (Circ J 2006; 70: 1184-1189)
\end{abstract}

Key Words: Cell transplantation; Myocardial infarction; Skeletal myoblasts; Ventricular remodeling

$\mathbf{M}$ yocardial infarction (MI) often leads to congestive heart failure because there is no functional regeneration of the infarcted myocardium due to the remaining cardiomyocytes being unable to re-enter the cell cycle. Within the past several years, cellular therapy (cardiomyoplasty) has emerged as a novel concept for endstage heart failure after MI! Experimental studies have used different cell types, such as cardiomyocytes,, 3 skeletal myoblasts, ${ }^{4-15}$ fibroblasts, embryonic stem cells ${ }^{16}$ and bone marrow stem cells ${ }^{14,17-19}$ Of these, skeletal myoblast transplantation appears to be more relevant from a clinical point of view because skeletal myoblasts can be obtained by routine muscle biopsy from heart failure patients, and then transplanted autologously without the risk of graft rejection. Several clinical trials are underway to determine whether autologous skeletal myoblast transplantation is an effective and safe therapeutic strategy for severe heart failure due to MI, and some groups have demonstrated autologous skeletal myoblast survival in the human heart? $20-24$

We recently reported that a large number of neonatal skeletal myoblasts fully replace the infarcted myocardium associated with reverse left ventricular (LV) remodeling in

(Received April 18, 2006; revised manuscript received June 1, 2006; accepted June 21, 2006)

Department of Cardiovascular Surgery, Graduate School of Medicine, *School of Health Sciences, Faculty of Medicine, Kyoto University, Kyoto and **Department of Cardiovascular Surgery, National Hospital Organization, Nagara Medical Center, Gifu, Japan

Mailing address: Masashi Komeda, MD, Department of Cardiovascular Surgery, Graduate School of Medicine, Kyoto University, 54 Kawaharacho, Shogoin, Sakyo-ku, Kyoto 606-8507, Japan. E-mail: komelab@kuhp.kyoto-u.ac.jp a rat MI model. ${ }^{4}$ Because it is necessary to transplant a large number of myoblasts to replace the infarcted myocardium, the procedure has a risk of tissue overgrowth or massive cell death. Indeed, Reinecke and Murry have demonstrated a risk of tissue overgrowth that sometimes distorts the ventricular contour, when myoblasts are transplanted as a bolus.$^{10}$ One goal for cell transplantation is to secure a sufficient graft cell mass without tumor-like overgrowth. We hypothesized that repeated skeletal myoblast implantation is a feasible and effective cell delivery method for full replacement of diseased myocardium.

Thus, in this study, using a rat coronary ligation model the effects of repeated implantation of skeletal myoblasts were compared with those of single bolus transplantation in terms of the dimensional and functional improvement in the left ventricle, as well as the volume of transplanted myoblasts.

\section{Methods}

\section{Experimental Animals}

Male syngeneic Lewis rats weighing 250-290g were used in this study. All experiments were performed in accordance with the guidelines for Animal Experiments at Kyoto University which confirms to the law of "Guide for the Care and Use of Laboratory Animals".

\section{Skeletal Myoblast Isolation and Cell Labeling}

Skeletal muscles were harvested from the lower limbs of syngeneic Lewis neonatal rats (2-3 days old), and minced into small pieces with scissors. To release the skeletal myoblasts, the minced muscles were enzymatically digested in 

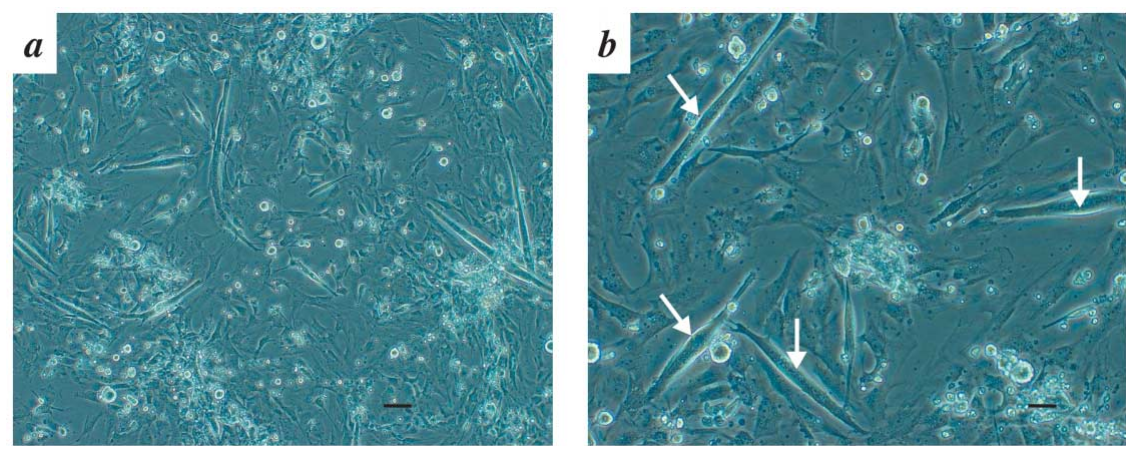

Fig 1. Representative cultured skeletal myoblasts from syngeneic Lewis neonatal rats at approximately $90 \%$ confluence. (a) $\times 100$, bar $=40 \mu \mathrm{m}$; (b) $\times 400$, bar $=10 \mu \mathrm{m}$. Arrows indicate multinucleated myotubes.

Dulbecco's modified eagle medium (DMEM) (Gibco Laboratory, Life Technologies, Grand Island, NY, USA) containing $0.35 \%$ trypsin (Gibco, Invitrogen Corporation, Grand Island, NY, USA) and $0.07 \%$ collagenase (Worthington, Lakewood, NJ, USA) at $37^{\circ} \mathrm{C}$ for $30 \mathrm{~min}$. The enzyme reaction was inactivated by the addition of DMEM containing 10\% fetal bovine serum. The cell suspension was filtered to remove undigested tissue elements, with a centrifugation at $500 \mathrm{G}$ for $5 \mathrm{~min}$. Skeletal myoblasts were purified by preplating for $40 \mathrm{~min}$ before plating on $60-\mathrm{mm}$ polystyrene tissue culture dishes, and kept in an incubator at $37^{\circ} \mathrm{C}$ in humid air with $5 \% \mathrm{CO}_{2}$. Passaging of cultures was carried out to maintain less than $70 \%$ density to avoid myogenic differentiation at higher densities. The cultured cells were harvested using $0.05 \%$ trypsin/ethylenediamine-tetraacetic acid (Gibco, Invitrogen Corporation), and kept on ice until transplantation. We transplanted the cultured skeletal myoblasts following in vitro expansion. We used cultured cells up to 3 passages and myoblasts were grown at less than $70 \%$ confluence to prevent premature differentiation. Indeed, we could observe myotubes when cells were grown more than $70 \%$ confluence (Fig 1 ). The $6 \times 10^{6}$ or $18 \times 10^{6}$ skeletal myoblasts were injected intramyocardially into the infarcted area with a volume of $0.1 \mathrm{ml}$. To separately identify each myoblast injection in group I, cell membranes of myoblasts used for the second and third implantation were labeled with PKH26 red fluorescent cell linker kit (Sigma Chemical Co, St Louis, MO, USA) and PKH 2 Green Fluorescent Cell Linker Kit (Sigma Chemical Co), respectively? 3

\section{Model}

Male Lewis rats were anesthetized with ethyl ether gas, and anesthesia was maintained with $0.8-1.5 \%$ isoflurane after intratracheal intubation. Through a left thoracotomy, the proximal part of the left anterior descending coronary artery (LAD) was ligated with a 6-0 polypropylene (Ethicon, Inc, Somerville, NJ, USA). ST-segment elevation on the electrocardiogram and color changes in the LV wall were observed in all rats.

\section{Experimental Groups}

Four weeks after the LAD ligation, rats with a moderatesized infarction were assigned to 3 treatment groups. In group I $(n=10)$, skeletal myoblasts $\left(6 \times 10^{6}\right)$ were subepicardially implanted in the infarct area at 0,2 and 4 weeks. In group II $(n=10)$, myoblast transplantation $\left(18 \times 10^{6}\right)$ was performed at 0 week, and culture medium $(0.1 \mathrm{ml})$ was injected at 2 and 4 weeks. In group III (control, $n=10)$ culture medium $(0.1 \mathrm{ml})$ was injected 3 times at 0,2 and 4 weeks. Thus, all rats had 3 thoracotomies for the myocardial injec- tions.

\section{Echocardiography}

Rats were anesthetized as described earlier. Left ventricular dimensions and functions were serially evaluated just before transplantation, and followed up 2, 4 and 8 weeks later. Transthoracic images were recorded using an ultrasound machine (Model 21380A with HP SONOS 5500 imaging, Agilent Technologies, Andover, MA, USA) equipped with a $12 \mathrm{MHz}$ phased-array transducer. A 2dimensional short-axis view of the LV was obtained at the level of the papillary muscles, and M-mode images were then recorded on the same plane for the measurement of the LV end-diastolic and end-systolic dimensions (LVDd and LVDs, respectively). Fractional area change (FAC) and the percentage of akinetic endocardial length to the whole LV endocardial circumference (AL) were also calculated from the same short-axis view. All measurements were performed in a blind fashion according to the American Society for Echocardiology, and averaged over 3 consecutive cardiac cycles.

\section{Cardiac Catheterization}

At 8 weeks after the first treatment, a $2 \mathrm{~F}$ micromanometer-tipped catheter (Millar Instruments Inc, Houston, TX, USA) was inserted through the right carotid artery into the $\mathrm{LV}$, and a $3 \mathrm{~F}$ occlusion balloon catheter (Fogarty, USA) through the right femoral vein into the inferior vena cava (IVC). LV pressure and its first-time derivative $(\mathrm{dP} / \mathrm{dt})$ were continuously monitored using a multiple recording system. LV end-systolic volume was calculated from the LVDs by the cube formula. During IVC occlusion with the balloon, LV pressure waveforms and M-mode tracings were simultaneously recorded. The end-systolic pressurevolume points obtained from echocardiography and cardiac catheterization were subjected to least squared linear regression, and the LV maximal time-varying elastance (Ees) was calculated as the slope of the fitted line. The time constant of isovolumic relaxation (Tau) was derived from the LV pressure curve.

\section{Histology}

Immediately after cardiac catheterization, all rats were killed, and the excised hearts were fixed with $4 \%$ buffered paraformaldehyde. The specimens were paraffin-embedded, and the whole LV was sectioned in $3 \mu \mathrm{m}$ thickness at $100 \mu \mathrm{m}$ intervals along the short axis. Histological sections were stained for fast skeletal myosin heavy chain (FSMHC) to detect the implanted myoblasts.,5 After deparaffinization, endogenous peroxidase activity was quenched by incubating with $0.1 \% \mathrm{NaN} 3$ and $0.3 \% \mathrm{H}_{2} \mathrm{O}_{2}$ in deionized 
Table 1 Echocardiographic Data at Pretreatment and at 2, 4 and 8 Weeks After First Transplantation in 4 Groups

\begin{tabular}{|c|c|c|c|c|}
\hline & Pre treatment & 2 weeks & 4 weeks & 8 weeks \\
\hline \multicolumn{5}{|l|}{$L V D d(\mathrm{~cm})$} \\
\hline Group I & $0.96 \pm 0.02$ & $0.95 \pm 0.02$ & $0.93 \pm 0.02$ & $0.90 \pm 0.02 * \dagger$ \\
\hline Group II & $0.98 \pm 0.03$ & $0.95 \pm 0.03$ & $0.94 \pm 0.02$ & $0.93 \pm 0.02^{\dagger}$ \\
\hline Group III & $0.96 \pm 0.03$ & $0.97 \pm 0.03$ & $0.99 \pm 0.02$ & $1.00 \pm 0.02 *$ \\
\hline \multicolumn{5}{|l|}{ LVDs (cm) } \\
\hline Group I & $0.77 \pm 0.03$ & $0.75 \pm 0.02$ & $0.73 \pm 0.01$ & $0.70 \pm 0.02 * \dagger$ \\
\hline Group II & $0.78 \pm 0.02$ & $0.74 \pm 0.02$ & $0.73 \pm 0.02$ & $0.73 \pm 0.02 * \dagger$ \\
\hline Group III & $0.76 \pm 0.02$ & $0.77 \pm 0.03$ & $0.79 \pm 0.01$ & $0.81 \pm 0.01$ \\
\hline \multicolumn{5}{|l|}{$F A C(\mathrm{~cm})$} \\
\hline Group I & $35.0 \pm 1.6$ & $37.6 \pm 1.5$ & $39.6 \pm 1.1 * \dagger$ & $41.3 \pm 1.8^{* \dagger}$ \\
\hline Group II & $35.2 \pm 2.2$ & $40.3 \pm 2.2$ & $40.5 \pm 2.6^{* \dagger}$ & $40.8 \pm 2.0^{* \dagger}$ \\
\hline Group III & $35.5 \pm 1.9$ & $35.5 \pm 1.8$ & $34.3 \pm 1.7$ & $33.9 \pm 1.4$ \\
\hline \multicolumn{5}{|l|}{$A L(\%)$} \\
\hline Group I & $29.3 \pm 1.5$ & $26.4 \pm 1.7$ & $23.6 \pm 1.5^{*, \dagger}$ & $22.3 \pm 1.8^{* \dagger}$ \\
\hline Group II & $30.6 \pm 1.6$ & $23.7 \pm 1.6$ & $23.9 \pm 1.5^{* \dagger}$ & $23.6 \pm 1.8^{* \dagger}$ \\
\hline Group III & $30.2 \pm 1.4$ & $30.5 \pm 1.1$ & $30.4 \pm 1.0$ & $30.5 \pm 1.2$ \\
\hline
\end{tabular}

Values are mean $\pm S E M$.

$L V D d$, left ventricular end-diastolic dimension; LVDs, left ventricular end-systolic dimension; FAC, fractional area change; $A L$, the percentage of akinetic endocardial length to the whole left ventricular endocardial circumference.

${ }^{*} p<0.05$ vs pretreatment values in the same group, ${ }^{\dagger} p<0.05$ vs values in group III.

and distilled water following antigen retrieval with boiled citrate buffer ( $\mathrm{pH}$ 6.0). Normal rabbit serum (Histofine SAB-PO (M) Kit, Nichirei, Tokyo, Japan) was added, followed by endogenous avidin-biotin blocking (Blocking Kit SP-2001, Vector Laboratories Inc, Burlingame, CA, USA). The sections were then incubated with anti-FSMHC antibody (1:400, clone My 32, Sigma-Aldorich Inc, St Louis, $\mathrm{MO}$, USA) at $4{ }^{\circ} \mathrm{C}$ overnight. Goat anti-mouse IgG antibody and peroxidase-labeled streptavidin (Histofine SAB-PO (M) Kit, Nichirei) were sequentially applied to visualize blue fluorescence at $450 \mathrm{~nm}$ wave length. The sections processed using PKH26 and PKH2 kits to observe red and green fluorescence, respectively, 3,5 Before each transplantation, we observed the previously transplanted area by echocardiography and injected myoblasts into a different region within the scar.

\section{Estimation of Donor-Derived Cell Volume}

Donor-derived muscle cell volume was calculated for each heart specimen using Simpson's formula described previously4,5 Briefly, the brown area occupied with FSMHCpositive cells $\left(\mathrm{S}, \mathrm{mm}^{2}\right)$ was calculated using an image analysis software (Scion Image Beta 4.02 Win, Scion Corporation, Frederick, MD, USA), and the engrafted volume $\left(\mathrm{V}, \mathrm{mm}^{3}\right)$ in each heart was calculated using the following formula: $V=0.1 \times \sum\left(S_{1}+S_{2}+\cdot \cdot+S_{n}\right) \quad(n=$ the number of sections that include FSMHC-positive areas).

\section{Statistical Analysis}

All data are expressed as the mean \pm SEM. Comparisons of echocardiography data among the groups were performed by 2-way repeated measures analysis of variance (ANOVA) including time, group, and group-by-time interaction terms. Comparisons of cardiac catheterization data and estimated graft volumes among the groups were conducted by 1-way factorial ANOVA. If significance was recognized for the group effect or the group-by-time interaction, post hoc comparisons among the groups or among the groups at each time point were performed, and if significance was found for the time effect or the group-by-time interaction, post hoc comparisons among the time points in each group were made, when appropriate, using Fisher's protected least significant difference method. All statistical analyses were performed using computer software (StatView for Windows version 5.0, SAS Institute Inc, Cary, NC, USA). A probability value $<0.05$ was considered statistically significant.

\section{Results}

The mortality rate due to coronary artery ligation and the infarct size were both similar to those in our previous studies. One rat in the 18-million bolus myoblast implantation group died immediately after injection, probably because of misinjection of myoblasts into the LV cavity with subsequent embolization. It can be technically difficult to inject a large number of myoblasts with a high viscosity into the thin scarred LV wall of rats; data for this animal were excluded from subsequent analysis.

\section{Echocardiography}

Echocardiographic data are shown in Table 1. There were no differences among the 3 groups regarding pretreatment LVDd, LVDs and FAC. In groups I and II, LVDd were significantly smaller at 4 and 8 weeks compared with pretreatment baseline values. In contrast, in group III, LVDd gradually increased and was significantly larger at 8 weeks than at baseline. As a result, at 8 weeks, LVDd in groups I and II was significantly smaller than that in group III. The changes in LVDs were directionally similar to those in LVDd. FAC in groups I and II gradually increased and reached a statistical significance at 4 and 8 weeks. Meanwhile, FAC in group III slightly decreased throughout the experiment period. At 4 and 8 weeks, FAC in groups I and II was significantly larger than that in group III. AL in groups I and II was significantly decreased by myoblast transplantation at 4 and 8 weeks after treatment. At that time, AL in groups I and II was significantly smaller than that in group III.

\section{Cardiac Catheterization}

Ees in group I was significantly higher than those in groups II and III $(0.86 \pm 0.05$ vs $0.70 \pm 0.04,0.42 \pm 0.03 \mathrm{mmHg} /$ $\mu 1$, respectively). Ees in group II was also significantly higher than that in Group III (Fig 2). Tau in groups I and II was significantly lower than that in Group III $(16.7 \pm 0.9$, 
$\operatorname{Ees}(\mathrm{mmHg} / \mu \mathrm{l})$

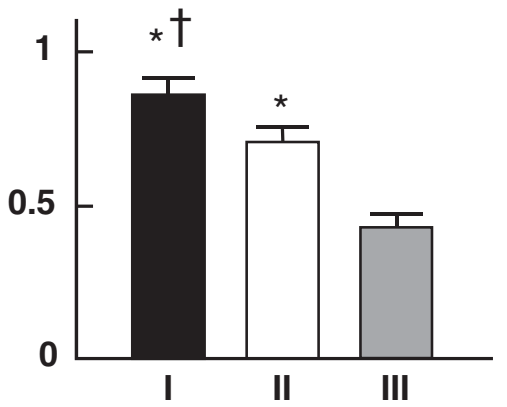

Tau (ms)

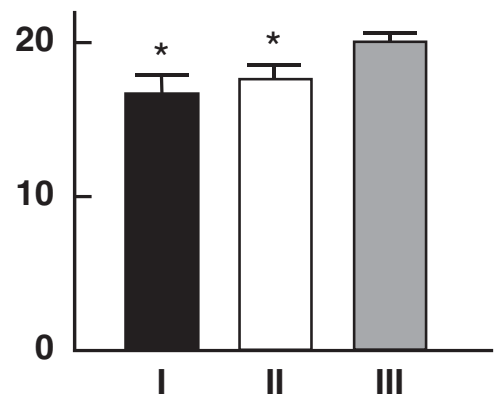

Fig 2. Comparisons of end-systolic elastance (Ees, Left panel) and time constant of isovolumic relaxation (Tau, Right panel) among the 3 groups. Group I, repeated myoblast transplantation; group II, bolus myoblast transplantation; group III, culture medium injection. ${ }^{*} \mathrm{p}<0.05$ vs group III, ${ }^{\dagger}<0.05$ vs group II.
$17.4 \pm 0.9$ vs $20.0 \pm 0.7 \mathrm{~ms}$, respectively). There was no significant difference between groups I and II (Fig 2).

\section{Histology}

At 4 weeks after the third transplantation in group I, myoblasts from the second transplantation were clearly visualized with red fluorescence (Fig 3a) and those of the third transplantation were clearly depicted with green fluorescence (Fig 3b) under fluorescence microscopy. All transplanted skeletal myoblasts in group I showed blue fluorescence (Figs 3c,d). In group I, transplanted myoblasts at each time point survived in the infarcted area. Furthermore, donor cells from the multiple transplantations occupied most of the infarcted area with nearly normal wall thickness (Figs 3e,f). Initially transplanted myoblasts spread throughout the infarcted area, whereas subsequently transplanted myoblasts were concentrated at 2 different locations in the infarcted area, usually adhering to the initially transplanted myoblasts. In group I, donor cells showing positive FSMHC extended over the entire infarcted area, whereas in group II, donor cells were confined to a limited area in the scar (Fig 4). Donor-derived skeletal muscle volume in group I was significantly larger than that in group II (13.6 \pm 1.0 vs $\left.8.0 \pm 0.8 \mathrm{~mm}^{3}\right)$ (Fig 5).

\section{Discussion}

The major findings of the present study are summarized.

(1) Repeated implantation significantly improved LV systolic and diastolic functions concurrent with infarct size reduction.

(2) Repeated skeletal myoblast transplantation provided a significantly larger engrafted volume and LV contractility compared with bolus transplantation of the same total number of myoblasts. In a previous study in a chronic rat infarction model, we showed that a
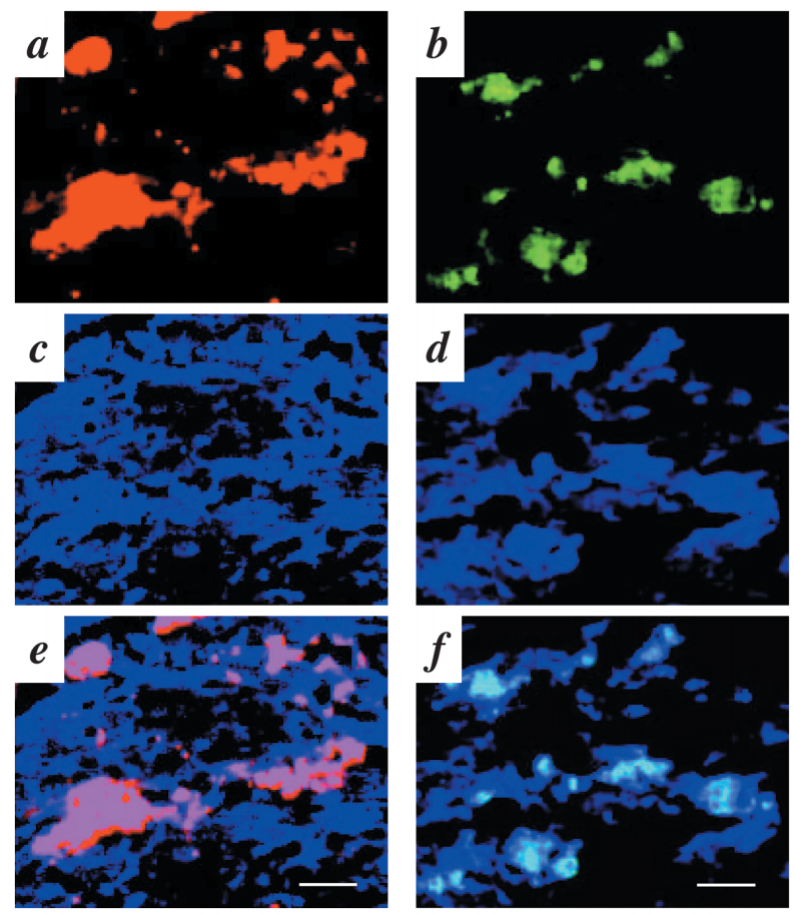

Fig 3. Representative immunofluorescent staining of transplanted skeletal myoblasts in the repeated transplantation group $(\times 400)$. Bars $=10 \mu \mathrm{m}$. (a), (c) and (e) are the same section, which is the case in (b), (d) and (f). (a) Red fluorescence indicates second group of transplanted myoblasts. (b) Green fluorescence indicates the third group of transplanted myoblasts. (c) and (d) Blue fluorescence indicates all transplanted myoblasts positive for fast skeletal myosin heavy chain. (e) Merged picture of (a) and (c). (f) Merged picture of (b) and (d). Subsequently transplanted myoblasts adhered to the initially transplanted myoblasts.
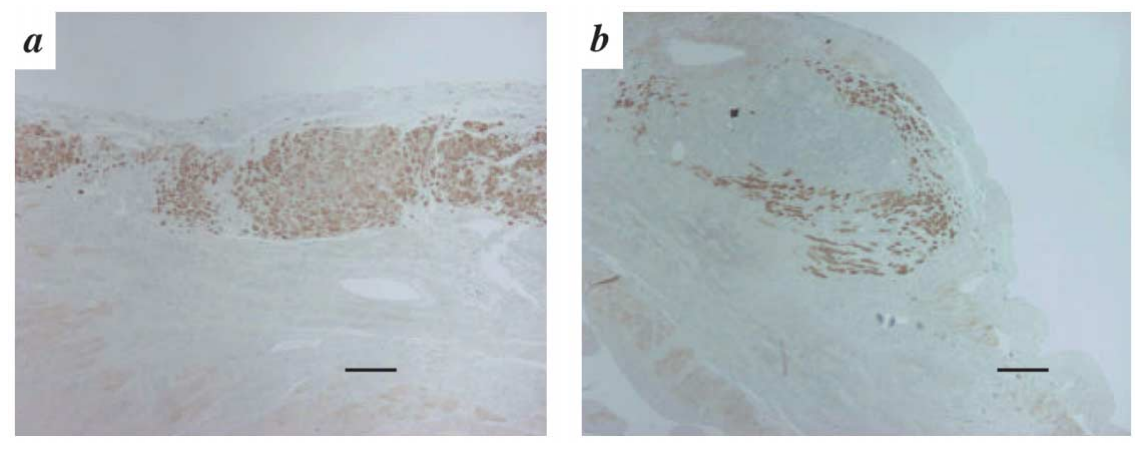

Fig 4. Expression of fast skeletal myosin heavy chain in the transplanted myoblasts (brown). (a) and (b) are representative pictures from groups I and II, respectively $(\times 40)$. The engrafted volume was larger in the group I compared with group II. Bars = $40 \mu \mathrm{m}$. Group I, repeated myoblast transplantation; group II, bolus myoblast transplantation. 


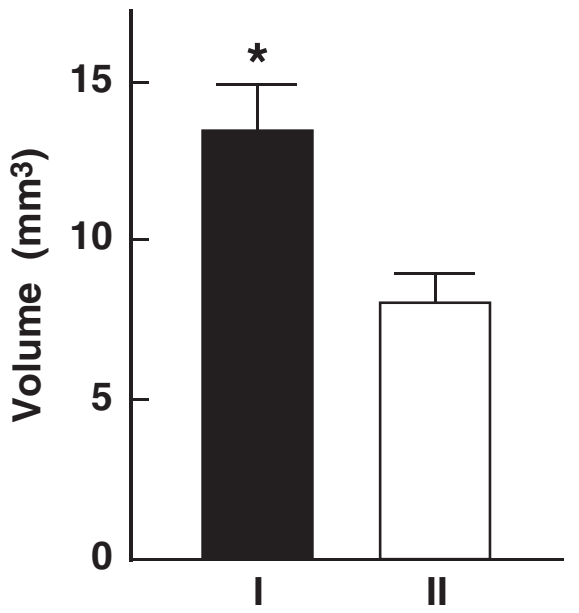

Fig 5. Estimated transplanted myoblast volume in groups I and II. Group I, repeated myoblast transplantation; group II, bolus myoblast transplantation. ${ }^{*} \mathrm{p}<0.0001$ vs group II.

large number of transplanted skeletal myoblasts replaced the infarcted myocardium with reverse LV remodeling. Histological findings in some of the hearts transplanted with large numbers of myoblasts had epi- or endocardial distortion and/or distension, although dimensional and fractional improvement was observed even in distorted hearts. Reinecke and Murry have demonstrated the risk of tissue overgrowth distorting the LV contour when a large number of myoblasts were transplanted as a single bolus? In our study, none of the LV undergoing 3 episodes of myoblast implantation demonstrated either distortion or distension. In future experiments, the most appropriate number of cells and episodes of skeletal myoblast transplantation should be determined.

Our findings confirm the results of our previous study; that is, the volume of skeletal myoblast-derived muscular tissue correlates with the extent of cardiac functional improvement? The decreased LV wall stress resulting from the increased wall thickness consisting of transplanted myoblasts may account for the improvement in cardiac performance. Thus, although more benefits may be expected from a larger volume of transplanted myoblasts, a bolus injection of myoblasts may lead to the ischemia in the center of the area of transplanted cells, which is the case in cardiomyocyte transplantation?,3 In contrast, repeated transplantation of a smaller volume of cells may have protected the myoblasts from ischemia-induced cell death. In the present study, although the engrafted myoblast volume in group I was significantly larger than that in group II, most indexes of cardiac function, except for Ees, were comparable between the 2 groups. This implies that Ees is the most sensitive indicator of myocardial intrinsic contractility, whereas wall motion is largely affected by preload and afterload of the LV.

In the clinical setting, a recent multicenter pilot study of autologous skeletal muscle transplantation has provided some evidence that this modality offers a potential therapeutic benefit for patients with end-stage heart disease ${ }^{20-24}$ Our present experimental study has demonstrated that repeated skeletal myoblast transplantation further improves cardiac function concurrent with reverse LV remodeling compared with a bolus injection of myoblasts. This pro- posed approach will be realized also in the clinical setting, using catheter-based, percutaneous skeletal myoblast transplantation via a novel needle-injection catheter ${ }^{23}$

\section{Study Limitations}

First, this study was performed in ischemic rat hearts and it is uncertain whether these findings can be applied to humans without further studies using large animals. Second, we did not stain junctional protein molecules. In our previous studies, 4,5 we were unable to demonstrate gap junction markers in the intercalated disks, and failed to demonstrate a connection between host myocardium and transplanted cells, which is common in most studies of skeletal myoblast transplantation. Third, quantitative analysis of angiogenesis was not performed because our aim was to investigate the surviving engrafted cell volume. Fourth, we did not use immunosuppressive agents because they would have increased the risk of infection. We performed all procedures under sterile conditions and there were no deaths from infection. Finally, further study using autologous skeletal myoblasts instead of neonatal skeletal myoblasts appears to be necessary when this model is considered for autologous myoblast transplantation in mature subjects.

In conclusion, there are no previous reports describing the effects of repeated skeletal myoblasts implantation, although single bolus transplantation of a large number of myoblasts has been reported ${ }^{10-12}$ Repeated myoblast transplantation showed a significantly larger engrafted volume without distorting the LV contour, and this cell delivery method also produced better elastic and/or contractile ability of the LV, both of which have been reported as important factors for successful myoblast transplantation.-8 Although our method may provide a clue for the full replacement of the infarcted myocardium with myoblast transplantation, further studies in large animal models are required to improve the reproducibility and to monitor long-term effects.

\section{Acknowledgments}

We would like to express our gratitude to Mrs Fumiyo Kataoka for her expert technical assistance, and acknowledge the meticulous assistance of Miss Yukiko Okumura during the preparation of this manuscript.

\section{References}

1. Fukuda K. Progress in myocardial regeneration and cell transplantation. Circ J 2005; 69: 1431-1446.

2. Li RK, Mickle DAG, Weisel RD, Zhang J, Mohabeer MK. In vivo survival and function of transplanted rat cardiomyocytes. Circ Res 1996; 78: $283-288$.

3. Sakakibara Y, Nishimura K, Tambara K, Yamamoto M, Lu F, Tabata $\mathrm{Y}$, et al. Prevascularization with gelatin microspheres containing basic fibroblast growth factor enhances the benefits of cardiomyocyte transplantation. J Thorac Cardiovasc Surg 2002; 124: 50-56.

4. Tambara K, Sakakibara Y, Sakaguchi G, Lu F, Premaratne GU, Lin $\mathrm{X}$, et al. Transplanted skeletal myoblasts can fully replace the infarcted myocardium when they survive in the host in large numbers. Circulation 2003; 108: 259-263.

5. Tambara K, Premaratne GU, Sakaguchi G, Kanemitsu N, Lin X, Nakajima $\mathrm{H}$, et al. Administration of control-released hepatocyte growth factor enhances the efficacy of skeletal myoblast transplantation in rat infarcted hearts by greatly increasing both quantity and quality of the graft. Circulation 2005; 112: I-129-I-134.

6. Taylor DA, Atkins BZ, Hungspreugs P, Jones TR, Reedy MC, Hutcheson KA, et al. Regenerating functional myocardium: Improved performance after skeletal myoblast transplantation. Nat Med 1998; 4: $929-933$.

7. Atkins BZ, Hueman MT, Meuchel JM, Cottman MJ, Hutcheson KA, Taylor DA. Myogenic cell transplantation improves in vivo regional performance in infarcted rabbit myocardium. J Heart Lung Trans- 
plant 1999; 18: $1173-1180$.

8. Hutcheson KA, Atkins BZ, Hueman MT, Hopkins MB, Glower DD, Taylor DA. Comparison of benefits on myocardial performance of cellular cardiomyoplasty with skeletal myoblasts and fibroblasts. Cell Transplant 2000; 9: 359-368.

9. Borenstein N, Bruneval P, Hekmati M, Bovin C, Behr L, Pinset C, et al. Noncultured, autologous, skeletal muscle cells can successfully engraft into ovine myocardium. Circulation 2003; 107: 3088-3092.

10. Reinecke H, Murry CE. Transmural replacement of myocardium after skeletal myoblast grafting into the heart: Too much of a good thing? Cardiovascr Pathol 2000; 9: 337-344.

11. Koh GY, Klug MG, Soonpaa MH, Field LJ. Differentiation and long-term survival of c2c12 myoblast grafts in heart. J Clin Invest 1993; 92: $1548-1554$.

12. Pouzet B, Vilquin JT, Hagège AA, Scorsin M, Messas E, Fiszman $\mathrm{M}$, et al. Factors affecting functional outcome after autologous skeletal myoblast transplantation. Ann Thorac Surg 2001; 71: 844-851.

13. Jain M, DerSimonian H, Brenner DA, Ngoy S, Teller P, Edge ASB, et al. Cell therapy attenuates deleterious ventricular remodeling and improves cardiac performance after myocardial infarction. Circulation 2001; 103: 1920-1927.

14. Gaurita-Souza LC, Carvalho KAT, Rebelatto C, Senegaglia A, Hansen P, Furuta M, et al. Cell transplantation: Differential effects of myoblasts and mesenchymal stem cells. Int J Cardiol 2006 (in press).

15. Chazaud B, Hittinger L, Sonnet C, Champagne S, Corvoisier PL, Benhaiem-Sigaux N, et al. Endoventricular porcine autologous myoblast transplantation can be successfully achieved with minor mechanical cell damage. Cardiovasc Res 2003; 58: 444-450.

16. Klug MG, Soonpaa MH, Koh GY, Field LJ. Genetically selected car- diomyocytes from differentiating embryonic stem cells form stable intracardiac grafts. J Clin Invest 1996; 98: 216-224.

17. Wang A, Fan YQ, Li CL, He H, Sun Y, Li BJ. Human bone marrowderived mesenchymal stem cells transplanted into damaged rabbit heart to improve heart function. J Zhejiang Univ Sci B6 2005; 4: $242-248$.

18. Orlic D, Kajstura J, Chimenti S, Jakoniuk I, Anderson SM, Li B, et al. Bone marrow cells regenerate infarcted myocardium. Nature 2001; 410: $701-705$.

19. Xu X, Xu Z, Xu Y, Cui G. Selective down-regulation of extracellular matrix gene expression by bone marrow derived stem cell transplantation into infarcted myocardium. Circ J 2005; 69: 1275-1283.

20. Menasché $\mathrm{P}$, Hagège AA, Vilquin JT, Desnos M, Abergel E, Pouzet B, et al. Autologous skeletal myoblast transplantation for severe postinfarction left ventricular dysfunction. J Am Coll Cardiol 2003; 41: $1078-1083$.

21. Dib N, Michler RE, Pagani FD, Wright S, Kereiakes DJ, Lengerich $\mathrm{R}$, et al. Safety and feasibility of autologous myoblast transplantation in patients with ischemic cardiomyopathy: Four-year follow-up. Circulation 2005; 112: 1748-1755.

22. Sim EKW, Jiang S, Ye L, Lim YL, Ooi OC, Haider KH. Skeletal myoblast transplant in heart failure. J Card Surg 2003; 18: 319-327.

23. Smits PC, van Geuns RJM, Poldermans D, Bountioukos M, Onderwater EEM, Lee CH, et al. Catheter-based intramyocardial injection of autologous skeletal myoblasts as a primary treatment of ischemic heart failure. Cell Transplant 2003; 42: 2063-2069.

24. Pagani FD, DerSimonian H, Zawadzka A, Wetzel K, Edge ASB, Jocoby DB, et al. Autologous skeletal myoblasts transplanted to ischemia-damaged myocardium in humans. J Am Coll Cardiol 2003; 41: 879-888. 